\title{
Primary Intraventricular Hemorrhage in Pediatric Patients: Causes, Characteristics, and Outcomes
}

\section{Rui Guo}

Sichuan University West China Hospital

\section{Chaofeng Fan}

Sichuan University West China Hospital

\section{Ruiqi Chen}

Sichuan University West China Hospital

\section{Zhiyuan Yu}

Sichuan University West China Hospital

\section{Rui Tian}

Sichuan University West China Hospital

\section{Chao You}

Sichuan University West China Hospital

Lu Ma ( $\sim$ huaxi_malu@163.com )

Sichuan University West China Hospital

\section{Research Article}

Keywords: Primary intraventricular hemorrhage; pediatric; surgery; vascular disorders

Posted Date: February 5th, 2019

DOI: https://doi.org/10.21203/rs.2.296/v1

License: (c) (i) This work is licensed under a Creative Commons Attribution 4.0 International License.

Read Full License

Version of Record: A version of this preprint was published at World Neurosurgery on January 1st, 2020. See the published version at https://doi.org/10.1016/j.wneu.2019.08.145. 


\section{Abstract}

Background: Primary intraventricular hemorrhage is a rare type of stroke and little is known its causes, characteristics and outcomes in children.

Methods: We retrospectively analyzed the clinical characteristics of patients aged one month to 21 years during seven consecutive years with primary intraventricular hemorrhage. Primary intraventricular hemorrhage was defined as bleeding confined to the ventricular system without parenchymal or subarachnoid hemorrhage involved.

Results: Of the included eighteen patients, $55.6 \%$ were female, and the mean age was $13.8 \pm 6.0$ years. The most common symptoms on presentation were headache (77.8\%) and vomiting (33.3\%). Fifteen patients (83.3\%) were diagnosed with known etiologies, including arteriovenous malformations (66.7\%), Moyamoya disease (11.1\%), and aneurysms (5.6\%). Idiopathic PIVH was diagnosed in only 3 patients (16.7\%). Fifteen patients (83.3\%) underwent surgical intervention, and 3 patients $(16.7 \%)$ received conservative treatment. Four patients $(28.6 \%)$ had an unfavorable outcome at discharge and unfavorable was observed in 3 patients (16.7\%) at the 3-month follow-up. Higher Graeb score was associated with an unfavorable outcome both in short-time and long-time follow-up.

Conclusions: Most primary intraventricular hemorrhage patients were diagnosed with arteriovenous malformations in the pediatric population. Specific surgical treatment of underlying etiologies should be required to increase clinical improvement. The children with higher Graeb score at admission tended to have poor early and late outcome.

Keywords: Primary intraventricular hemorrhage; pediatric; surgery; vascular disorders.

\section{Background}

Primary intraventricular hemorrhage (PIVH) is rare, constituting less than $9 \%$ of intracerebral hemorrhage (ICH).[1-4] PIVH is intraventricular hemorrhage with bleeding confined to the ventricular system. [1] The etiology of PIVH is complex, which can be caused by a variety of underlying vascular disorders.[5-7] Furthermore, the guidelines for PIVH management is absent. These conditions make this kind of hemorrhagic stroke a challenge for neurosurgeons and neurologists.

Although previous reports have explored the clinical features and management of PIVH,[1-7] they focused on this disease in adult patients. Relatively little is known about PIVH in pediatric patients. Moreover, the past two decades have seen a remarkably increasing burden on pediatric stroke,[8] a better understanding of PIVH in the pediatric patients becomes more important. Our study tried to explore the causes, clinical features, treatment and outcome of PIVH in the children.

\section{Methods}




\section{Study population}

We reviewed 18 consecutive PIVH patients aged one month to 21 years who were treated in West China Hospital from 2010 to 2016 . All the parents of these children or themselves (if they were $\geq 18$ years old) gave their informed consent in this study. Our study was approved by the ethics committee of West China Hospital, Sichuan University. PIVH was diagnosed after neurologists or neurosurgeons reviewed initial computed tomography (CT) scans, which showed intraventricular hemorrhage without intraparenchymal or subarachnoid hemorrhage. Patients with a history of head trauma were excluded from the present study.

\section{Data Collection}

Baseline information was collected by reviewing medical records, including demographic data, presentation, treatment, and neurologic outcome. Computed tomography angiography (CTA) or digital subtraction angiography (DSA) as performed for all patients as routine to screen possible underlying vascular lesions. The imaging data were independently assessed by two neurologists blinded to the patients' clinical information. The intraventricular blood volume was evaluated by a Graeb score.[9] Modified Rankin Scale (mRS) score was used to assess the neurologic status of the patients at discharge. Follow-up information for all the included patients was obtained by telephone interview or outpatient review. Patients were followed up at 3 months after discharge. We inquired about the neurologic status (mRS), which was dichotomized as favorable (mRS 0-2) and unfavorable (mRS 3-6) functional outcomes.

\section{Statistical Analysis}

Statistical analyses were performed using SPSS software (version 24.0; IBM Corp.). Continuous variables were reported as the mean + standard deviation, and categorical variables were expressed as the absolute number combined with valid percentage. The Student $t$ test or Mann-Whitney $U$ test was used to analyze differences in the mean or median of continuous variables. Categorical variables were analyzed by the chi-square test. Besides, logistic regression analysis was performed to assess independent factors for short-time and long-time outcomes. A p values $\leq 0.05$ were considered statistically significant.

\section{Results}

\section{Demographic Data and Clinical Manifestations}

Between 2010 and 2016, a total of 18 consecutive pediatric PIVH patients were treated in West China hospital. The demographic data and clinical characteristics of the patients are summarized in Table 1. There were 10 female (55.6\%) and 8 male (44.4\%) patients, and the sex ratio was 1:0.8 female-male. The mean age was $13.8 \pm 6.0$ years. The most common initial manifestation was headache $(n=14,77.8 \%)$, followed by vomiting $(n=6,33.3 \%)$ and disturbance of consciousness $(n=4,22.2 \%)$. The mean GCS score of these 18 pediatric patients was $12.3 \pm 4.0$, and the mean Graeb score was $3.4 \pm 2.4$ at 
admission. All the patients underwent computed tomography angiography (CTA) or/and digital subtraction angiography (DSA) at admission. According to preoperative CTA or/and DSA results, 15 patients (83.3\%) were diagnosed with underlying vascular disorders, including arteriovenous malformation (AVM) in 12 patients (66.7\%), Moyamoya disease in 2 patients (11.1\%) and aneurysm in 61 patients (91.0\%). The angiography for the other 3 patients was negative, who were diagnosed with idiopathic PIVH. The average length of hospital stay was $16.2 \pm 8.2$ days for all these 18 patients.

\section{Treatment}

Surgical findings and clinical outcomes are summarized in Table 2. All patients in our series were presented to an integrated ward of neurological diseases which consists of neurosurgeons, endovascular neurologists, and neuroradiologists. In general, 15 patients (83.3\%) received surgical intervention, and 3 patients (16.7\%) underwent conservative treatment in the present study. For the 5 patients with acute hydrocephalus, external ventricular drainage (EVD) was performed in 4 patients and microsurgical evacuation of the hematoma was performed in the other patient with ventricular hemorrhage cast. All the 12 patients with AVM received at least one kind of surgical intervention, including resection, endovascular embolization and stereotactic radiation. For the 2 patients with Moyamoya disease, one with acute hydrocephalus received EVD followed by revascularization surgery after the hemorrhage was totally absorbed. The other one declined any surgical intervention. And the patients with aneurysm underwent EVD and endovascular embolization.

\section{Clinical Outcomes}

The functional outcomes at discharge and 3-month follow-up are shown in Table 3,4 and Figure 1. The patient with aneurysm died in hospital. From discharge to 3 months, neurologic status was improved in 9 patients $(50.0 \%)$ and remained stable in 7 cases $(46.7 \%)$. One patient with idiopathic PIVH died at one month after discharge because of rebleeding. At discharge, favorable outcomes ( $m R S \leq 2)$ were observed in 14 cases (77.8\%), and the 3-month follow-up, the number increased to 15 patients (83.4\%).

The 3-month functional outcome of pediatric PIVH patients after surgical intervention improved but it had no statistical significance compared with discharge (discharge mRS score 1.93 vs 3-month mRS score $1.73 ; p=0.67)$. However, the condition in patients with acute hydrocephalus showed opposite trend (discharge mRS score 2.40 vs 3-month mRS score 3.40; $p=0.44$ ). For patient with GCS $\leq 8$, the functional status deteriorated with no statistical significance (discharge mRS score 2.2 vs 3 -month mRS score 3.00; $p=0.58$ ). The neurological outcome of idiopathic PIVH patients seemed to decline in terms of the mean early and late mRS score (discharge mRS score 0.33 vs 3-month mRS score $2.33, p=0.42$ ). We also dichotomized our patients into two groups: patients with small hematoma volume (Graeb score $\leq 6$, $n=16$ ) and those with large hematoma volume (Graeb score $\geq 7, n=2$ ). For the overall patients, patients in the small-volume group harbored significantly better outcome than those with large volume both at discharge and 3-month follow-up ( $p=0.002$ and $p=0.01$, respectively). Furthermore, we analyzed the characteristics of patients with poor outcome. We found that higher Graeb score was associated with an 
unfavorable outcome both in short-time and long-time follow-up ( $p=0.04$ and $p=0.02$, respectively) (Table 3 and Table 4).

\section{Discussion}

$\mathrm{PIVH}$ is a unique subtype of intracerebral hemorrhage characterized by hemorrhage confined to the ventricular system. Because of the overall rarity of PIVH, related studies in pediatric population remain even scarce, which has made identifying consistent characteristics of PIVH difficult.[1-7] The present study analyzed 18 cases of pediatric PIVH treated at our center (Table 1) between 2010 and 2016. Several unique characteristics were found regarding etiology, treatment and clinical outcome. The results of our study may help achieve a better understanding of pediatric PIVH.

Interestingly, sex distribution varied significantly in previous adult studies.[6, $7,10,11]$ Among the patients in study of Marti-Fabregas et al. and Srivastava et al., the majority were male (38.5\% and $37.03 \%$, respectively), $[7,10]$ whereas some other studies by Hameed et al. and Giray et al. showed an opposite trend, in which there were more males $(60.0 \%$ and $58.3 \%$, respectively) than females. $[6,11]$ Our studies reported a more balanced distribution between boys and girls. In our cohort, the sex ratio was malefemale $0.8: 1$, which demonstrated no obvious difference in the sex distribution among pediatric PIVH patients. Research findings have proved that gender difference may have affected the incidence and physiopathologic process of stroke.[12,13] However, the impact of gender on pediatric PIVH is yet to be determined in further studies.

Previous studies reported various etiological constitution of PIVH.[1-7, 10, 11, 14] In most studies, the most common possible etiology for adult PIVH was hypertension (from $50.0 \%$ to $80 \%$ ). $[3,5,6,11]$

Flint et al. reviewed $99 \mathrm{PIVH}$ patients who underwent a catheter angiogram in 15 case series, presented that AVM seemed to be the most common (54.5\%) underlying vascular abnormality in adult PIVH, followed by aneurysm (39.4\%), and Moyamoya disease (5.1\%).[15] Our study presented quite a similar constitution: AVM in $66.7 \%$ of the 18 pediatric patients, Moyamoya disease in $11.1 \%$, aneurysm in $5.6 \%$. Only 3 children in our study could not be identified with vascular disorders, which was inconsistent with elder patients in previous reports.[1-5, 10,11]

To date, because of a lack of related research, the appropriate method to achieve maximum benefit for pediatric PIVH patients remain unknown. An external ventricular drainage (EVD) remains the current primary method for PIVH with obstructive hydrocephalus. In our study, $83.3 \%$ of pediatric patients with PIVH underwent a surgical procedure, and 4 of the 5 patients $(90.0 \%)$ with acute hydrocephalus received EVD. In previous reports among elder population, this percentage was much lower.[1-7, 10,11, 14, 15] The mass effect of intraventricular hemorrhage (IVH) and acute obstructive hydrocephalus caused by IVH may be basic pathophysiologic factors in PIVH, which can elevate intracranial pressure (ICP) and decrease cerebral perfusion.[16] EVD is assumed to drain cerebrospinal fluid out of ventricular system, reducing ICP. However, EVD alone is inadequate and is often occluded with blood clots.[17] Besides. EVD cannot change the blood clot resolution rate after IVH, failing to relieve communicating hydrocephalus. 
$[18,19]$ Studies have explored the effectiveness and safety of the concomitant use of antifibrinolytics to decrease the complications of EVD. [20,21] However, the debate on which method is better for pediatric $\mathrm{PIVH}$ is continuing.

Previously reported case series in adult PIVH had relatively unfavorable clinical outcomes, with inhospital mortality ranging from $13.3 \%$ to $42.0 \%$. However, in contrast, only 1 patient (5.6\%) in the present pediatric cohort did not survive at discharge, and another patient with idiopathic PIVH died one month after discharge because of rebleeding. In our study, favorable outcomes (mRS $\leq 2$ ) were observed in 14 cases $(77.8 \%)$ at discharge, and at the 3-month follow-up, the number increased to 15 patients (83.4\%). Our pediatric PIVH patients had better outcome compared to previous adult studies. This might be partly thanks to the improved diagnostic ability, allowing the early recognition of benign cases. Furthermore, rapid access to neurological intensive care and acute management strategies in our hospital may help these pediatric PIVH patients achieve better clinical outcomes.

There are several limitations in our study. First, the patient number was relatively small, with only 18 pediatric patients. Second, this study was a single-center retrospective cohort which lacked randomization between groups. Besides, this study was a chart review, in which some study patients could have been missed in our identification process. Furthermore, because of the absence of guidelines for PIVH, our treatment strategies, might have been applied with controversy. A further multicenter clinical trial with a larger sample size is warranted to provide better insights of pediatric $\mathrm{PIVH}$.

\section{Conclusions}

PIVH in pediatric patients has distinctive features compared with adult patients. Most primary intraventricular hemorrhage patients were diagnosed with arteriovenous malformations in the pediatric patients. Specific surgical treatment of underlying etiologies should be required to increase clinical improvement. higher Graeb score was associated with an unfavorable outcome both in short-time and long-time follow-up. However, a multi-centered study, with larger sample size is still necessary to provide better insights of pediatric PIVH.

\section{Abbreviations}

AVM: arteriovenous malformation; CT: computed tomography; CTA = computed tomography angiography; DSA: digital subtraction angiography; EVD: external ventricular drainage; GCS: Glasgow Coma Scale; ICH: intracerebral hemorrhage; IVH: intraventricular hemorrhage; mRS: modified Rankin scale; PIVH: primary intraventricular hemorrhage

\section{Declarations}

\section{Acknowledgements}

The authors thank the patients who were involved in this study. 


\section{Funding}

This work was supported by no funding.

\section{Availability of data and materials}

The datasets used and/or analyzed during the current study are available from the corresponding author on reasonable request.

\section{Authors' contributions}

RG contributed to data analysis, manuscript drafting and revising. CFF and RQC contributed to data collection, and interpretation of the data. ZYY and RT contributed to data analysis and interpretation. CY contributed to study design and manuscript revising. LM contributed to study design, manuscript drafting and revising. All authors read and approved the final manuscript.

\section{Ethics approval and consent to participate}

Written informed consent was obtained from patients or themselves खif they were $\geq 18$ years old. This study was approved by the Ethics Committee of West China Hospital.

\section{Consent for publication}

Not applicable.

\section{Competing interests}

The authors declare no competing interests.

\section{References}

1. Darby DG, Donnan GA, Saling MA, Walsh KW, Bladin PF. Primary intraventricular hemorrhage: clinical and neuropsychological findings in a prospective stroke series. Neurology. 1988, 38(1):68-75.

2. Jayakumar PN, Taly AB, Bhavani UR, Arya BY, Nagaraja D. Prognosis in solitary intraventricular haemorrhage. Clinical and computed tomographic observations. Acta Neurol Scand. 1989, 80(1):1-5.

3. Angelopoulos M, Gupta SR, Azat Kia B. Primary intraventricular hemorrhage in adults: clinical features, risk factors, and outcome. Surg Neurol. 1995, 44(5):433-6; discussion 437.

4. Passero S, Ulivelli M, Reale . Primary intraventricular haemorrhage in adults. Acta Neurol Scand. 2002, 105(2):115-9.

5. Kiymaz N, Demir O, Cirak B. Is external ventricular drainage useful in primary intraventricular hemorrhages? Adv Ther. 2005, 22(5):447-52.

6. Giray S, Sen O, Sarica FB, Tufan K, Karatas M, Goksel BK, Yerdelen D, Cekinmez M, Can U.

Spontaneous primary intraventricular hemorrhage in adults: clinical data, etiology and outcome. Turk 
Neurosurg. 2009, 19(4):338-44.

7. Srivastava T, Sannegowda RB, Satija V, Jain RS, Tejwani S, Mathur T: Primary intraventricular hemorrhage. clinical features, risk factors, etiology, and yield of diagnostic cerebral angiography. Neurol India. 2014, 62(2):144-8.

8. Amlie-Lefond C, Rivkin MJ, Friedman NR, Bernard TJ, Dowling MM, deVeber G. The Way Forward: Challenges and Opportunities in Pediatric Stroke. Pediatr Neurol. 2016, 56:3-7.

9. Graeb DA, Robertson WD, Lapointe JS, Nugent RA, Harrison PB. Computed tomographic diagnosis of intraventricular hemorrhage. Etiology and prognosis. Radiology. 1982, 143(1):91-6.

10. Marti-Fabregas J, Piles S, Guardia E, Marti-Vilalta JL. Spontaneous primary intraventricular hemorrhage: clinical data, etiology and outcome. J Neurol. 1999, 246(4):287-91.

11. Hameed B, Khealani BA, Mozzafar T, Wasay M. Prognostic indicators in patients with primary intraventricular haemorrhage. J Pak Med Assoc. 2005, 55(8):315-7.

12. Vaartjes I, Reitsma JB, Berger-van Sijl M, Bots ML. Gender differences in mortality after hospital admission for stroke. Cerebrovasc Dis. 2009, 28(6):564-71.

13. Wagner I, Volbers B, Kloska S, Doerfler A, Schwab S, Staykov D. Sex differences in perihemorrhagic edema evolution after spontaneous intracerebral hemorrhage. Eur J Neurol. 2012, 19(11):1477-81.

14. Arboix A, Garcia-Eroles L, Vicens A, Oliveres M, Massons J. Spontaneous primary intraventricular hemorrhage: clinical features and early outcome. ISRN Neurol. 2012, 2012:498303.

15. Flint AC, Roebken A, Singh V. Primary intraventricular hemorrhage: yield of diagnostic angiography and clinical outcome. Neurocrit Care. 2008, 8(3):330-6.

16. Ziai WC, Tuhrim S, Lane K, McBee N, Lees K, Dawson J, Butcher K, Vespa P, Wright DW, Keyl PM et al. A multicenter, randomized, double-blinded, placebo-controlled phase III study of Clot Lysis Evaluation of Accelerated Resolution of Intraventricular Hemorrhage (CLEAR III). Int J Stroke. 2014, 9(4):536542.

17. Carhuapoma JR. Thrombolytic therapy after intraventricular hemorrhage: do we know enough? J Neurol Sci. 2002, 202(1-2):1-3.

18. Naff NJ, Williams MA, Rigamonti D, Keyl PM, Hanley DF. Blood clot resolution in human cerebrospinal fluid: evidence of first-order kinetics. Neurosurgery 2001, 49(3):614-9; discussion 619621.

19. Ngo QN, Ranger A, Singh RN, Kornecki A, Seabrook JA, Fraser DD. External ventricular drains in pediatric patients. Pediatr Crit Care Med. 2009, 10(3):346-51.

20. Staykov D, Huttner HB, Struffert T, Ganslandt O, Doerfler A, Schwab S, Bardutzky J. Intraventricular fibrinolysis and lumbar drainage for ventricular hemorrhage. Stroke. 2009, 40(10):3275-80.

21. Kumar K, Demeria DD, Verma A. Recombinant tissue plasminogen activator in the treatment of intraventricular hemorrhage secondary to periventricular arteriovenous malformation before surgery: case report. Neurosurgery. 2003, 52(4):964-968; discussion 968-969. 


\section{Tables}

Table 1 - Baseline characteristics and outcomes in pediatric patients with PIVH

Characteristics Value

Total number of patients $\quad 18$

Age, years (mean $\pm \mathrm{SD}) \quad 13.8 \pm 6.0$

Gender, n (\%)

Male

$8(44.4)$

Female

$10(55.6)$

Initial symptoms, n (\%)

Headache

$14(77.8)$

Vomiting

6 (33.3)

Disturbance of consciousness

$4(22.2)$

GCS score, mean \pm SD

$12.3 \pm 4.0$

Graeb score, mean \pm SD

$3.4 \pm 2.4$

Acute hydrocephalus, n (\%)

$5(27.8)$

Etiological causes, n (\%)

Moyamoya disease

$2(11.1)$

AVM

$12(66.7)$

Aneurysm

$1(5.6)$

Idiopathic

$3(16.7)$

Length of hospital stay

$16.2 \pm 8.2$

Table 2 - Comparison of treatment and observation cohorts 


\begin{tabular}{lccl}
\hline Characteristic & Surgical treatment & Conservative treatment & P Value \\
& $(\mathrm{n}=15)$ & $(\mathrm{n}=3)$ & \\
\hline Gender & 6 & 2 & 0.40 \\
Male & 9 & 1 & \\
Female & $14.3 \pm 6.0$ & $11.0 \pm 6.2$ & 0.39 \\
Mean age(yrs) & & & \\
Initial symptoms & 11 & 3 & 0.31 \\
Headache & 5 & 1 & 0.73 \\
Vomiting & 4 & 0 & 0.31 \\
Disturbance of consciousness & $11.7 \pm 4.2$ & $15.0 \pm 0.0$ & $0.009^{*}$ \\
GCS score & $3.2 \pm 2.5$ & 0 & 0.47 \\
Graeb score & 5 & & 0.24 \\
Acute hydrocephalus, & & 1 & 0.18 \\
Etiological causes & 1 & 0 & $0.007^{*}$ \\
Moyamoya disease & 12 & 0 & 0.65 \\
AVM & 1 & 2 & $0.01^{*}$ \\
Aneurysm & 1 & $0.7 \pm 0.6$ & 0.19 \\
Idiopathic & $1.9 \pm 1.5$ & $0.7 \pm 0.6$ & 0.87 \\
mRs score at discharge & $1.7 \pm 2.1$ & & \\
mRs score at 3 months & & & \\
\hline
\end{tabular}

* $\mathrm{P}<0.05$

Table 3 - Favorable (mRS Scores 0-2) and unfavorable (mRS Scores 3-6) outcome at hospital discharge

\begin{tabular}{|c|c|c|c|c|}
\hline Variables & 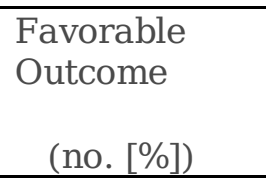 & $\begin{array}{l}\text { Unfavorable } \\
\text { Outcome } \\
\text { (no. [\%]) }\end{array}$ & $\begin{array}{c}\mathrm{P} \\
\text { value }\end{array}$ & OR $(95 \% \mathrm{CI})$ \\
\hline NO. of patients & 14 & 4 & & \\
\hline Gender & & & 0.80 & $0.75(0.08-6.96)$ \\
\hline Male & $6(75.0)$ & $2(25.0)$ & & \\
\hline Female & $8(80.0)$ & $2(20.0)$ & & \\
\hline Mean age(yrs) & $13.3 \pm 6.3$ & $15.5 \pm 4.8$ & 0.53 & $1.07(0.87-1.32)$ \\
\hline \multicolumn{5}{|l|}{ Initial symptoms } \\
\hline Headache & $12(85.7)$ & $2(14.3)$ & 0.13 & $6.00(0.51-7.67)$ \\
\hline $\begin{array}{l}\text { Disturbance of } \\
\text { consciousness }\end{array}$ & $2(50.0)$ & $2(50.0)$ & 0.13 & $0.17(0.01-1.96)$ \\
\hline GCS score & $12.9 \pm 3.5$ & $10.3 \pm 5.5$ & 0.26 & $0.85(0.65-1.12)$ \\
\hline Graeb score & $3.2 \pm 2.3$ & $4.0 \pm 2.9$ & 0.04 & $1.52(1.12-1.84)$ \\
\hline Acute hydrocephalus, n (\%) & $3(60.0)$ & $2(40.0)$ & 0.26 & $0.27(0.03-2.83)$ \\
\hline \multicolumn{5}{|l|}{ Etiological causes, n (\%) } \\
\hline AVM & $9(75)$ & $3(25.0)$ & 0.69 & $0.60(0.05-7.41)$ \\
\hline Length of hospital stay & $15.1 \pm 6.6$ & $20.0 \pm 12.9$ & 0.30 & $1.08(0.94-1.23)$ \\
\hline
\end{tabular}

Table 4 - Favorable and unfavorable functional outcome at the 3-month follow-up 


\begin{tabular}{|c|c|c|c|c|}
\hline Variables & 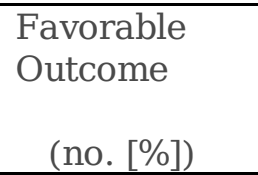 & $\begin{array}{l}\text { Unfavorable } \\
\text { Outcome } \\
\text { (no. [\%]) }\end{array}$ & $\begin{array}{c}\mathrm{P} \\
\text { value }\end{array}$ & OR $(95 \%$ CI $)$ \\
\hline NO. of patients & 15 & 3 & & \\
\hline Gender & & & 0.40 & $0.33(0.02-4.55)$ \\
\hline Male & $6(75.0)$ & $2(25.0)$ & & \\
\hline Female & $9(90.0)$ & $1(10.0)$ & & \\
\hline Mean age(yrs) & $14.3 \pm 5.8$ & $11.0 \pm 7.5$ & 0.40 & $0.91(0.73-1.13)$ \\
\hline Initial symptoms & & & & \\
\hline Headache & 13(92.9) & $1(7.1)$ & 0.05 & $\begin{array}{l}13.00(0.77- \\
20.01)\end{array}$ \\
\hline $\begin{array}{l}\text { Disturbance of } \\
\text { consciousness }\end{array}$ & $2(50.0)$ & $2(50.0)$ & 0.08 & $0.08(0.01-1.30)$ \\
\hline GCS score & $12.9 \pm 3.6$ & $9.3 \pm 5.1$ & 0.17 & $0.81(0.60-1.10)$ \\
\hline Graeb score & $3.3 \pm 2.4$ & $3.7 \pm 2.9$ & 0.03 & $1.56(1.23-1.86)$ \\
\hline Etiological causes, n (\%) & & & & \\
\hline AVM & $11(91.7)$ & $1(8.3)$ & 0.18 & $5.50(0.39-7.57)$ \\
\hline Idiopathic & $2(66.7)$ & 1(33.3) & 0.40 & $0.31(0.02-5.19)$ \\
\hline Length of hospital stay & $16.1 \pm 8.9$ & $16.3 \pm 4.9$ & 0.97 & $1.00(0.86-1.17)$ \\
\hline
\end{tabular}

\section{Figures}


At discharge (\%)

At 3 months (\%)

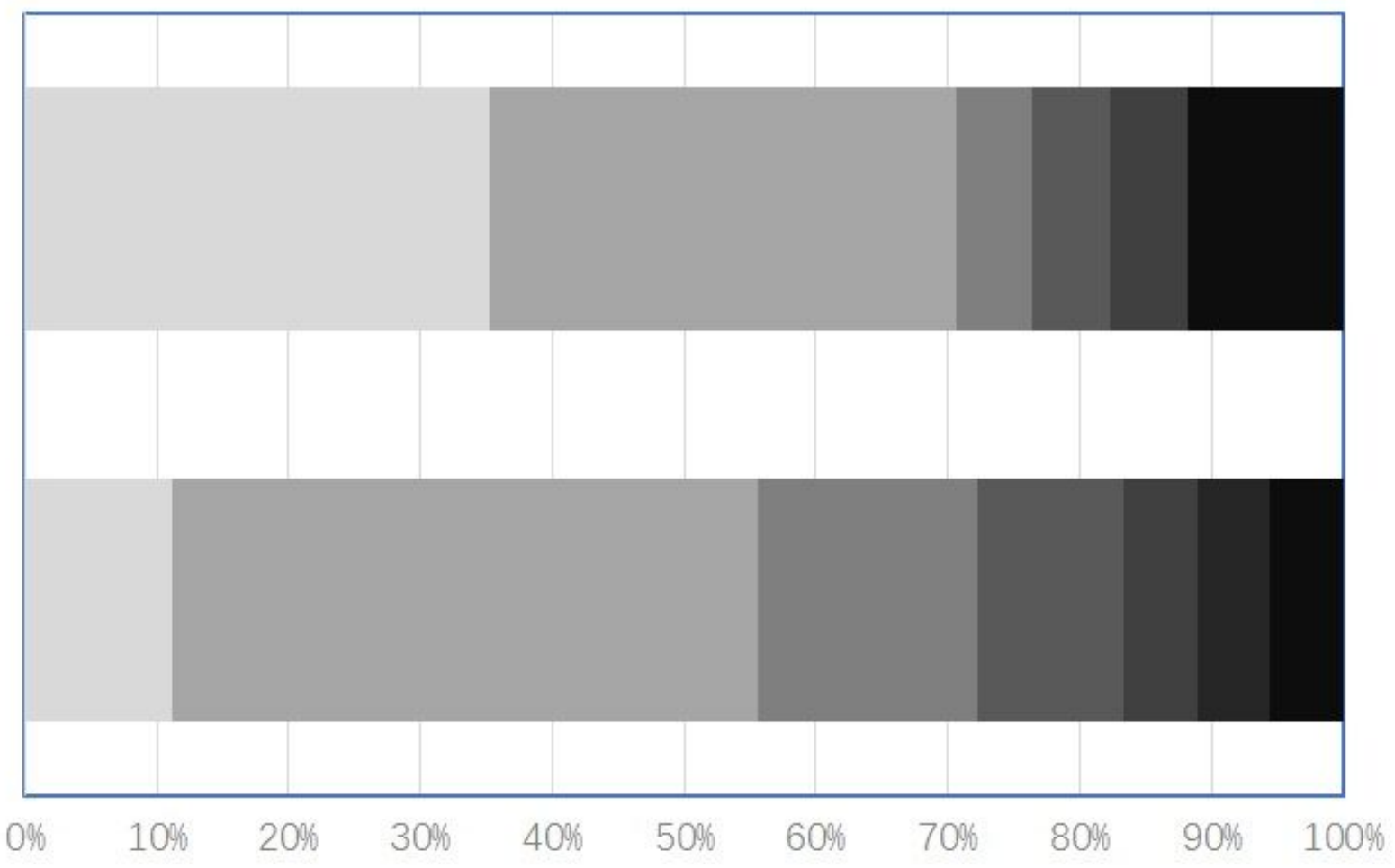

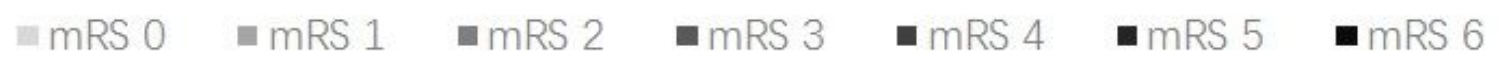

\section{Figure 1}

Functional outcome according to the mRS at discharge and 3-month follow-up.

\section{Supplementary Files}

This is a list of supplementary files associated with this preprint. Click to download.

- supplement1.doc 